\title{
Entre liberté et contrôle, les privat-docents et l'enseignement de la médecine à Genève entre 1876 et $1917^{*}$
}

Philip Rieder

\section{Summary}

The University of Geneva was founded in 1876 with two sets of teachers established as competitive entities: the salaried professors and the nonsalaried privat-docents. The ease with which physicians obtained the title of privat-docent, a series of abuses of that status and the professors' desire to manage teaching at all levels, resulted in a conflictual situation. The subsequent drive of professors to gain control over all teaching reveals tensions between the medical faculty and the political authorities, but also strains within the medical community itself. Ultimately, the result was the professors' leadership over the rest of the medical community.

\section{Résumé}

En 1876, la nouvelle Université de Genève compte deux corps enseignants organisés sur le principe de la concurrence, les enseignants salariés et les privat-docents. La cohabitation est difficile à la Faculté de médecine; la facilité d'accès au statut de privat-docent, les abus que certains privat-docents commettent ainsi que la volonté des professeurs de maîtriser l'enseignement en sont les raisons principales. Le combat que mènent les titulaires salariés

* Cet article émane d'un projet de recherche entrepris à l'Institut Louis-Jeantet d'histoire de la médecine (Genève) sur l'histoire de la Faculté de médecine de Genève. Il reprend et approfondit un exposé présenté à la réunion annuelle de la Société suisse d'histoire de la médecine et des sciences naturelles, en octobre 1995. Je remercie les participants de cette journée pour leurs commentaires critiques, ainsi que Mireille Lador, Joëlle Droux et Vincent Barras pour leurs remarques constructives sur des versions antérieures de ce travail.

Philip Rieder,Institut Louis Jeantet d'histoire de la médecine, Centre médical universitaire, Case postale, 1211 Genève 4 
pour gagner le contrôle de l'ensemble de l'enseignement sert de révélateur des tensions entre la Faculté et les pouvoirs politiques, ainsi que des enjeux propres au corps médical genevois.

L'histoire de l'enseignement de la médecine se réduit souvent à des données quantitatives relatives au nombre d'étudiants, aux locaux disponibles ou au mieux, à une chronologie des décisions administratives prises. Des étudiants suivent le cursus imposé, certains obtiennent le grade envisagé, d'autres échouent. Une autre façon d'aborder le sujet pourrait être celle qui tenterait de s'approcher de la réalité des cours, de la personnalité des enseignants. Comment saisir, par exemple, l'image du médecin qu'on cherche à former? Un moyen de parvenir à ces fins consiste à rechercher les problèmes qui se posaient à l'époque, à retrouver les enjeux tels qu'ils se présentaient aux acteurs. Cette approche nous a amené à nous pencher sur le statut des privatdocents à Genève, statut qui permet à de jeunes enseignants de tenter leur chance dans les auditoires, sans recevoir pour cela de salaire. A la fin du siècle, la Faculté nouvellement créée semblait mal placée pour mettre en cause cet «enseignement libre». Peu à peu, les professeurs prennent position pour affirmer leur désir de contrôler l'ensemble de l'enseignement, en motivant leur démarche par le souci de garantir le sérieux et la scientificité de la formation. Nous verrons qu'il s'agit là, en grande partie, de redéfinir et défendre une certaine idée du médecin qu'on compte former. D'autre part, le durcissement de la position de la Faculté nous semble réfléchir aussi son assurance croissante, consolidée par le nombre toujours plus important d'étudiants, et sur le plan corporatif, l'affermissement progressif du corps professoral dans son rôle dominant au sein du corps médical genevois.

L'introduction du statut de privat-docent est une des propositions les plus originales des articles du projet de loi, présenté au Grand Conseil en 1871, articles destinés à régir la future Université. Ce statut d'enseignant sans solde rappelle bien le statut de «professeur libre» qui existait déjà dans l'ancienne Académie, ou - et ici l'affiliation est plus nette encore - celui des Privat-Docenten dans les universités allemandes contemporaines. Pourtant, c'est l'amalgame des caractéristiques de ces deux statuts qui en fait l'originalité. L'absence d'épreuves d'habilitation rappelle - jusqu'aux lettres de la loi - celui des «professeurs libres» de l'ancienne Académie ${ }^{2}$; tandis que l'octroi du titre de privat-docent évoque le statut des universitaires allemands, admis dans le corps enseignant des universités germaniques moyen-

2 Comparer l'art. 83 de la Loi générale sur l'Instruction publique du 25 octobre 1848 avec l'art. 132 de la loi de 1872. 
nant la défense publique d'une thèse supplémentaire (habilitation) ${ }^{3}$. Dans le projet législatif genevois, le privat-docent - du moment où il possède un titre reconnu par l'Université - peut annoncer «dans les programmes, des cours libres et se servir, pour ces cours, des salles affectées à l'enseignement supérieur». La possibilité est même donnée au Département de l'Instruction publique (DIP) d'en faire bénéficier, «après avoir pris le préavis du Sénat Académique, (...) [les] personnes qui, par des publications, par un enseignement antérieur, par un examen, auront donné des preuves suffisantes de capacité». Une telle facilité d'accès à l'enseignement s'inscrit bien dans la logique de l'université que prône le professeur et député Carl Vogt, une université libérale largement inspirée par le «modèle allemand» ${ }^{4}$. Les articles concernant les privat-docents sont adoptés sans discussion, seule une proposition de Vogt vient libéraliser encore le texte définitif ${ }^{5}$. Le rôle de Vogt dans l'élaboration de la loi de 1872 et spécialement dans la conception de la nouvelle université est bien connu' ${ }^{6}$. Rappelons simplement que le monde universitaire y est régi par la loi de l'offre et de la demande. Dans cette logique, les privat-docents forment un corps enseignant qui doit concurrencer les professeurs; leurs activités garantissent la mise sur pied d'un enseignement gratuit - les privat-docents sont rétribués uniquement par le montant des inscriptions perçues pour leurs cours. A terme, ces enseignants doivent former la relève universitaire. La conjoncture locale ne manque pas de renforcer l'attrait du concept ${ }^{7} ; l^{\prime}$ 'idée de trouver des enseignants qu'il ne faut pas payer convient à tout le monde. En effet, si tous les membres du Grand Conseil sont d'accord sur l'utilité et le surcroît de prestige que constituerait la transformation de l'Académie en Université, ils sont nombreux à redouter la facture

3 Voir à ce sujet: BUSCH Alexander, «The Vicissitudes of the Privat-Docent» in Minerva,1963, vol. 1, p. 324.

4 Par exemple, VOGT Carl, «L'Université fédérale. Discours prononcé, le 5 mai 1870, à la séance annuelle de l'Institut National Genevois» in Bulletin de l'Institut National Genevois, t. xvi, 1870. Voir MARCACCI Marco, Histoire de l'Université de Genève, Genève, 1987, p.113. Carl Vogt n'est pas le seul à s'inspirer du modèle allemand, notamment pour ce qui concerne les privat-docents. L'idée semble même assez répandue, par exemple: RAMBERT Eugène, L'avenir de l'instruction supérieure dans la Suisse française, Genève-Bâle, 1869, p. 72.

5 Loi sur l'Instruction publique du 19 octobre 1872, Genève, 1872, art. 132. Vogt propose d'élargir l'accès au statut à des personnes ayant des compétences professionnelles reconnues.

6 Plus particulièrement: MARCACCI Marco, «Carl Vogt: le savoir, le pouvoir et le rôle de l'université», in Revue du Vieux Genève, n ${ }^{\circ}$ 17, 1987, pp. 44-52.

7 Un statut de privat-docent similaire, quoique moins libéral, est créé lors de la fondation de l'Université de Lausanne (1890). Le candidat doit adresser sa demande au Conseil d'Etat qui prend l'avis de l'Université, il y fait état de ses titres universitaires et de son expérience. Seuls les savants reconnus qui sont appelés par l'Université ou l'Etat en sont dispensés. S'il est retenu, le candidat est présenté aux étudiants de la faculté et doit faire une leçon d'ouverture (publiée à 200 exemplaires). Voir DELESSERT André, L'Université au défi, Lausanne, 1991, p. 205. 
qu'occasionnera la création de la Faculté de médecine ${ }^{8}$. Création indispensable, à l'époque, pour que la haute école genevoise porte le titre d'Université.

La campagne menée par les partisans du projet doit convaincre sur les capacités cliniques de l'Hôpital cantonal ${ }^{9}$, mais aussi sur le budget. Un des médecins les plus engagés en faveur du projet, le député radical Gustave Julliard, médecin et chirurgien à l'Hôpital cantonal, axe son argumentation budgétaire autour des privat-docents dans un rapport destiné au conseiller d'Etat Carteret.

«nous sommes sûrs d'avoir des privat-docents qui se chargeront de donner des cours jusque dans les branches les plus spéciales. Le fait n'est pas douteux dans une ville où il y a autant de médecins désireux de travailler au bien de leur pays et de la science et dans des positions qui leur permettent les loisirs et le travail de cabinet.»

Il continue même en suggérant l'intérêt que peut avoir cette institution dans le devenir de la Faculté; elle permettrait que les jeunes «professeurs se fassent connaître et se désignent au choix des autorités qui ainsi pourra avoir lieu en connaissance de cause $»^{10}$. Cette argumentation ne manque pas d'exercer un grand attrait sur les membres du Grand Conseil. Par ailleurs, l'accès ainsi donné aux «médecins en ville» est un moyen de surseoir à l'opposition traditionnelle des médecins genevois à l'organisation d'un enseignement médical ${ }^{11}$. Grâce au statut de privat-docent, tout médecin qui le désire peut s'associer à l'enseignement universitaire. C'est ainsi qu'à son ouverture (1876) la nouvelle Faculté comprend, outre 12 chaires principales, des cours donnés gratuitement par des privat-docents. Il s'agit d'une véritable libéralisation de l'enseignement. Les privat-docents sont intégrés au corps professoral sans pour autant être contrôlés par l'Université; leurs cours sont annoncés dans le programme des cours et affichés sur une grille horaire qui regroupe tous les enseignements; ils peuvent fonctionner comme jurés aux examens et ont une voie consultative dans les instances universitaires. L'incidence de cette structure sur la marche de la Faculté de médecine est difficile à soupeser. Les titulaires de charges d'enseignement sont soumis à la loi de l'offre et de la demande; ils doivent supporter la concurrence de toute personne diplômée qui se présente. Dans ce cadre, le rapport entre l'ensei-

8 Ces problèmes budgétaires ont failli faire capoter définitivement le «projet université»; le 2 septembre 1872, Carl Vogt lui-même estimait la réalisation trop coûteuse. Mémorial du Grand Conseil 1872, t. 3, pp. 1843-1844.

9 Ce qui est réalisé essentiellement par la brochure de Gustave JULLIARD, Notice sur l'Hôpital Cantonal, Lausanne, 1870.

10 JULLIARD Gustave, «Rapport de Julliard aux autorités, 10 septembre 1873» in La Faculté de Médecine de Genève 1876-1976, Genève, 1978, p. 14.

11 GAUTIER Léon, La médecine à Genève, Genève, 1906, pp. 336-337. Cette réticence était motivée par le désagrément de voir un médecin s'élever au-dessus des autres. 
gnement des professeurs et celui des privat-docents serait intéressant à étudier. Pour mieux cerner les enjeux, nous avons cherché à réunir les données éparpillées dans les registres des organes universitaires.

\section{Le privat-docent au quotidien (1876-1885)}

Dans le projet proposé par Julliard évoqué ci-dessus, seuls trois cours sont attribués aux privat-docents ${ }^{12}$. Les autres champs que ceux-ci devaient assurer sont vagues; ils «se chargeront de donner des cours jusque dans les branches les plus spéciales» ${ }^{13}$. Les premiers semestres donneront raison à Julliard et verront un grand nombre de cours de ce type annoncés dans le programme de la Faculté de médecine. Ils sont déjà 5 au semestre d'hiver 1875-1876, une année avant la création de la Faculté de médecine;1'année de l'ouverture (en 1876) le programme en compte $10^{14}$. Ce succès est considérable, comme l'attestent les données réunis par Billroth sur les universités allemandes - seuls 5 sur 15 institutions recensés en comptent plus à la même époque ${ }^{15}$. Les réalisations semblent bien répondre aux attentes des législateurs, et la progression des années suivantes confirme cette réussite - surtout en ce qui concerne la Faculté de médecine (voir fig. 3, p. 91). Elle s'explique sans doute partiellement (en médecine) par la facilité avec laquelle ce genre d'activité pouvait se concilier avec un travail en cabinet, mais aussi par le besoin des «médecins en ville», toujours plus nombreux, de se démarquer de leurs confrères par un titre universitaire. Pendant la période étudiée, il y a plus de privat-docents en médecine que dans toute autre faculté.

\section{a) Une politique d'encouragement}

Dans un premier temps et dans le contexte de l'effervescence suscitée par la mise en place de l'Université nouvellement créée, la tendance est de simplifier les procédures, d'accepter tous les candidats au titre de privat-docent. Cette politique semble refléter les inquiétudes des responsables de l'Université; ils comptent sur le concours des médecins établis en ville pour assurer la réussite de l'entreprise - sans savoir quelle sera l'importance de

12 Il s'agit de «pansements et bandages», «auscultation et percussion» et «analyse des urines».

13 Voir plus haut, note $\mathrm{n}^{\circ} 10$.

14 Programme des cours 1875-1876, Genève, 1875 ainsi que celui de l'année suivante. Le programme comporte nombre d'erreurs, mais est un indicateur relativement fidèle.

15 BILLROTH Theodor, Über das Lehren und Lernen der Medicinischen Wissenschaften an den Universitäten der Deutschen Nation, Wien, 1876, p. 256. 
leur collaboration ${ }^{16}$. Ainsi, en 1875 , le Recteur propose que le DIP dispense les autorités universitaires de reconsidérer chaque année les compétences de privat-docents déjà nommés, opération qu'il qualifie comme étant «oisive» ${ }^{17}$. Quelques mois avant l'inauguration, et malgré des réticences exprimées à la Faculté de médecine, les docteurs Guillot, Devrient et Geib se voient octroyer le statut de privat-docent ${ }^{18}$. Le Sénat accorde ce statut à tous les diplômés qui ont postulé - il est vrai que la loi ne prévoit pas le moyen d'effectuer un tri. Les réticences des professeurs de la Faculté de médecine, notamment par rapport aux trois médecins susmentionnés, se confirment dès les premiers mois d'enseignement. D'abord, la Faculté décide d'interpréter les articles de loi relatifs aux privat-docents de manière à ne reconnaître que les titres de ceux qui ont le droit de pratiquer ${ }^{19}$. Ensuite, l'opportunité de réviser le statut de privat-docent est discutée à plusieurs reprises, dès les premières séances de la Faculté de médecine. Et finalement, au moment du début des cours, les professeurs de médecine refusent de mettre à la disposition des privatdocents le «matériel», les instruments et collections de l'Université - le privat-docent dépend du bon vouloir de chaque enseignant pour l'enseignement clinique ${ }^{20}$. Cette volonté professorale de limiter la marge de manœuvre de ces enseignants, prévus dès l'origine comme «concurrents», s'explique de plusieurs manières. On pense d'abord à des préoccupations scientifiques, mais il ne faut pas négliger la perte évidente d'inscriptions d'étudiants (soit Frs 5.- par étudiant) au profit des privat-docents. En effet, tout comme les privat-docents, les professeurs de médecine touchent intégralement le produit des inscriptions versées par les étudiants qui suivent leurs cours - c'est le «casuel», lequel complète les salaires variables, mais souvent modestes des professeurs titulaires. Bientôt des doléances précises vont venir renforcer les réticences instinctives des professeurs, un certain nombre de privat-docents se targuant du titre, annonçant des cours, mais ne les donnant pas! Les noms

16 Cette inquiétude n'est pas sans fondement; le statut tout à fait comparable de professeur libre de l'ancienne Académie n'avait pas été un succès. Voir à ce propos le Rapport de la Commission chargée de réviser la loi sur l'Instruction Publique, lu au Grand Conseil le 8 juin 1864, Genève, 1864, p. 61.

17 Archives de l'Université, Dépôt du Seujet (par la suite «Seujet»), Pièces diverses et correspondance 1873-1878. La réponse positive du DIP figure dans la même liasse, sous la date du 11 novembre 1875, voir aussi Registre du Sénat, 23 novembre 1875.

18 Archives du Décanat (par la suite «Décanat»), Registre des séances de la Faculté de médecine, $\mathrm{n}^{\circ} 1,23$ mai et 30 mai 1876.

19 Ibid., n 1, p. 25. En conséquence, ceux qui n'ont pas cette autorisation et qui sont désireux de donner un enseignement doivent adresser une demande particulière au DIP.

$20 \mathrm{Ibid}$., $\mathrm{n}^{\circ} 1$, le 13 octobre 1876, p. 36. Les privat-docents demandent à pouvoir utiliser les instruments et les collections de la Faculté: «La Faculté ne peut leur reconnaître ce droit; mais il va sans dire que chaque professeur [a le droit] de prêter ce qu'il voudra, sous sa propre responsabilité». 
ne sont pas cités, mais en dépouillant les récépissés des inscriptions, il apparait que sur les 10 privat-docents annoncés au programme du premier semestre, seuls 5 ont des étudiants inscrits et sur ces 5, deux n'ont qu'un étudiant chacun $^{21}$ ! Dans les faits, la manière de procéder des privat-docents de la Faculté est pragmatique. Au début du semestre, ils affichent des listes sur lesquels les étudiants doivent venir s'inscrire. Après plusieurs semaines, s'ils en ont enregistré un nombre jugé suffisant, ils donnent leurs cours ${ }^{22}$. Bien sûr, les enseignants ne sont pas seuls responsables des auditoires vides. L'absence d'auditeurs est un risque qui fait déjà partie du système théorique: les privatdocents sans succès devraient renoncer à leur enseignement. Or, la loi ne les oblige pas à le faire et la pratique montre qu'ils ne le font pas. La répétition des plaintes laisse entendre que nombre de privat-docents profitent de l'ambiguïté du statut; ils profitent d'un titre qui ne leur coûte rien mais qui impressionne le public.

\section{b) Abus et première réglementation}

Ces plaintes répétées proviennent en grande partie de la Faculté de médecine, mais des voix protestent également dans d'autres facultés; très vite les autorités universitaires, d'entente avec le DIP (qui commence aussi à s'inquiéter ${ }^{23}$ ), cherchent à limiter les abus. En 1878, le DIP revient sur sa décision de 1875 et exige que les privat-docents redemandent chaque année l'autorisation d'enseigner ${ }^{24}$. Le 29 janvier 1879, la question est posée clairement au cours d'une séance du Sénat: «que faut-il faire pour restreindre les abus, par exemple de l'inscription de Privat-docenten qui ne donnent pas leurs cours?» La réponse trouvée par cette instance est de codifier les procédures d'accès au statut, d'exiger à la fois le préavis de la faculté concernée et la communication au Recteur, dans les 15 premiers jours du semestre, des cours qui n'ont pas lieu «sous peine de se voir refuser l'autorisation dans un autre semestre». C'est en substance la teneur du nouveau règlement de l'Université, approuvé par le Conseil d'Etat la même année ${ }^{25}$. Un règlement spécial,

21 Les cours qui marchent le mieux sont l'obstétrique, la gynécologie et une spécialité qui ne connaît pas d'enseignement officiel, la chirurgie dentaire.

22 On trouve ces indications dans le Bureau du Sénat (Seujet), 10 novembre 1899. Le Recteur se plaint que «la plus grande irrégularité règne parmi les Privat-docents en Médecine. Ils affichent une liste pour que les étudiants s'inscrivent, et commencent seulement quand ils jugent avoir suffisamment d'auditeurs. Sur la remarque de M. D'Espine que cela s'est toujours fait, le Bureau demande au département de fixer un délai de 15 jours après le commencement des cours pour le retard toléré.»

23 Bureau du Sénat, 26 janvier 1880.

24 Seujet, Pièces diverses et correspondance 1873-1878.

25 Règlement de l'Université de Genève, 1879. 
plus détaillé, sera mis en vigueur l'année suivante. Ce nouveau règlement entérine la décision prise par la Faculté de médecine de n'admettre comme privat-docents que des docteurs qui ont le droit de pratiquer à Genève ou en Suisse. L'Université s'assure également le droit, implicite dans la loi, d'examiner les titres des candidats pour juger de l'équivalence de leurs diplômes. Le seul moyen légal pour empêcher un candidat de devenir privatdocent est de ne pas reconnaître son diplôme. Deux autres articles ont pour but de réduire les abus: le titre de privat-docent ne peut être pris que par les enseignants «pendant la durée de leur enseignement effectif» (art.3) et le Sénat peut retirer l'autorisation d'enseigner, aux privat-docents «qui auraient commis une infraction au Règlements» (art. 7) ${ }^{26}$. D'autre part, la gestion des privat-docents est confiée au Bureau de l'Université; une organe plus efficace (composée de moins de membres) que le Sénat ${ }^{27}$. L'ensemble des ces mesures donne à l'Université les moyens de sanctionner les abus signalés précédemment. Pourtant, les faits montrent que le Sénat n'use pas de ce droit. Ce désinvestissement s'explique sans doute par une multitude de facteurs: le surmenage des professeurs, la croissance de leurs responsabilités administratives ou tout simplement, leur manque de motivation pour exercer une véritable police. Les professeurs de médecine font exception, une petite enquête sur les privat-docents nous permet de comprendre pourquoi.

\section{La Faculté de médecine et les privat-docents (1876-1885)}

Pour obtenir un reflet de l'importance des cours de privat-docents en médecine, nous avons dépouillé un certain nombre de cahiers d'inscriptions conservés dans les archives de l'Université. Cette source offre un reflet plutôt pessimiste de la réalité - le resquillage étant un problème pour les autorités universitaires. On peut néanmoins supposer sans trop de risques que les chiffres obtenus ont une valeur indicative. Dans l'ensemble, au sein de la Faculté de médecine, l'enseignement des privat-docents remplit au moins partiellement les attentes des législateurs. Avec les années, les enseignants de ce type sont toujours plus nombreux, donnant des cours théoriques et pratiques, com-

26 Règlement spécial pour les cours de privat-docents à l'Université, du 19 novembre 1880. Cette réglementation résulte de l'affaire Geib (voir plus bas, p. 13). Le DIP n'émet qu'une seule réserve (Bureau du Sénat, 18 juin 1880), en exigeant que «la clause sur les cours de privatdocent ne serait plus applicable dans le cas où un privat-docent ferait un cours sur les mêmes matières qu'un professeur ordinaire». Il faut maintenir une saine concurrence entre enseignants!

27 Loi sur l'Instruction publique du 19 octobre 1872, art. 136 et 137: le Bureau comprend le Recteur, le Vice-recteur, le Secrétaire (de l'Université) et les doyens de facultés. Le Sénat est formé par tous les professeurs ordinaires et honoraires. 
plémentaires à l'enseignement des professeurs. Les cours qui enregistrent le plus grand nombre d'inscriptions sont ceux annoncés par des assistants (ou anciens assistants) lesquels sont aussi privat-docents et qui complètent ou répètent l'enseignement des titulaires ${ }^{28}$, ce n'est pourtant pas la seule raison de succès. On peut signaler dans un premier temps l'intérêt que suscitent les cours (théoriques et pratiques) portant sur des domaines qui ne bénéficient pas encore d'un enseignement officiel. C'est le cas de la clinique infantile donné par le prof. D’Espine (pathologie interne). Après quatre ans de démarches inutiles pour obtenir un enseignement officiel, celui-ci annonce un cours libre, cours qu'il donnera d'ailleurs dans sa clinique privée ${ }^{29}$. Les maladies infantiles forment déjà une spécialisation reconnue, d'où le succès d'un enseignement qu'il donnera pendant 17 ans (1883-1899). D'autres docteurs proposent des cours cliniques concurrents, comme le Dr Martin, le médecin de l'Hôpital Gourgas («Maison des enfants malades»), qui assurera son enseignement de 1887 jusqu'à la fin de la période étudiée (1914). Dès qu'un semestre de clinique infantile est exigé par le règlement des examens fédéraux, D'Espine renonce à son enseignement «libre» ${ }^{30}$, mais d'autres continuent. C'est grâce à ces enseignants que les étudiants peuvent effectuer le semestre de clinique infantile dont ils ont besoin, et qui ne sera organisé officiellement qu'en 1907. Un autre domaine mal loti dans l'enseignement salarié de la Faculté de médecine est l'ophtalmologie. Jusqu'en 1891, l'enseignement est assuré par deux privat-docents, le Dr Barde et le Dr Haltenhoff; ces deux médecins donnent un enseignement théorique et pratique dans des cliniques privées ${ }^{31}$. L'enseignement clinique est particulièrement important puisqu'il figure au programme des examens fédéraux de médecine dès $1888^{32}$. Pourtant, Haltenhoff, le professeur extraordinaire nommé en 1891, ne parvient pas à convaincre le Conseil d'Etat de créer un service hospitalier. Une raison sans doute du succès des cours cliniques d'ophtalmologie proposés par les privat-docents. Les professeurs de clinique chirurgicale et médicale vont même jusqu'à aménager leur horaire d'enseignement pour que les étudiants puissent suivre celui de Barde ${ }^{33}$. L'exécutif genevois semble avoir profité de ce médecin, dont la valeur scientifique était reconnue (il

28 Ces donnés sont confirmés par le relevé des inscriptions par cours pour les premières années cf. GOSSE H. J., Marche de la Faculté de médecine dans les années 1882, 1883, 1884, Genève, 1886.

29 Décanat, Registre des séances de la Faculté de médecine, nº 1,12 mai 1885, pp. 248-249.

30 Règlement pour les examens fédéraux de médecine du 11 décembre 1899, art.51.C'est le moyen le plus sûr d'inciter le DIP à mettre sur pied l'infrastructure clinique nécessaire.

31 GOURFEIN David Elie, L'évolution de l'Ophtalmologie à Genève, Genève, 1921, p. 16.

32 Règlement pour les examens fédéraux de médecine du 19 mars 1888, art. 46.

33 Seujet, Diverses pièces et correspondance (1889-1894), lettres entre le prof. Revilliod, le Recteur et le DIP. 
était examinateur fédéral), pour retarder la mise en place d'un enseignement officiel ${ }^{34}$. Mentionnons ensuite une série de cours dont le succès ne peut s'expliquer que par la déficience ou par le nombre limité de professeurs titulaires. Ici, la gynécologie et l'obstétrique sont des exemples particulièrement intéressants. Le professeur Vulliet, chargé de la policlinique gynécologique, refuse de donner ses cours prétextant l'absence de moyens matériels. Son collègue, le professeur Vaucher, titulaire de la chaire de clinique obstétricale, a une mauvaise réputation (mauvais caractère) ${ }^{35}$, ses compétences sont même remises en question à la fin du siècle et il finit, suite à une pétition d'étudiants, par être mis en congé par le Conseil d'Etat. Il n'est pas étonnant, dans ces conditions, de voir un nombre considérable de cours très suivis donné dans ce domaine par des privat-docents.

La Faculté de médecine néglige ces succès pour se focaliser sur les abus qui se perpétuent et surtout, sur l'inefficacité de la répression de ces abus. Dans les instances universitaires, les mêmes plaintes reviennent, formulées la plupart du temps par des professeurs de la Faculté de médecine ${ }^{36}$. Il est vrai que l'administration des privat-docents par le Bureau du Sénat n'est pas à même de les rassurer. Tantôt cette instance exige que les délais pour annoncer les cours à venir soient respectés avec précision, tantôt des dérogations sont généreusement distribuées. Le plus souvent le Bureau se contente de gérer, de faire passer les dossiers des candidats au statut de privat-docent soit à la Faculté soit au DIP, de prendre note des cours qui sont annulés, des changements de titres - dans les quelques cas où il en est averti. La seule restriction importante imaginée par les autorités universitaires et les autorités politiques est l'interdiction générale, sauf dérogation, de donner des cours en dehors des bâtiments universitaires ${ }^{37}$. Cette restriction vise surtout les médecins qui disposent d'une clinique privée et qui pourraient être tentés d'y donner des cours cliniques; elle complète celle déjà prise par la Faculté elle-même, de contrôler l'accès des privat-docents aux laboratoires et aux

34 AEG, fonds DIP, Carton 1985 va 5.3.24, Médecine X. Le nouveau titulaire, le Dr Haltenhoff, dans son «Rapport sur l'enseignement de la Clinique Ophtalmologique à Genève», se plaint de la mauvaise volonté rencontrée dans la mise en place de cet enseignement.

$35 \mathrm{ROCH}$ M., «Libre histoire médicale des cent premières années de l'Hôpital cantonal, in Centième anniversaire de l'hôpital cantonal de Genève 1856-1956, Genève, 1956, pp. 90-91. Les registres de la Commission Administrative de l'Hôpital sont aussi éloquents à ce sujet.

36 Par exemple, le 21 mai 1886, au Bureau du Sénat, la question des privat-docents est abordée. «A propos M. Gosse remarque que quelques docteurs se sont fait inscrire depuis plusieurs années pour des cours libres qu'ils n'ont pas faits sans même avertir le doyen, et demande que l'on avise à supprimer cet abus lorsqu'on discutera à nouveau les règlements.» De nouveau, dix ans plus tard, le 16 mai 1896, Julliard «attire l'attention du Bureau sur le grand nombre de Privat-docents inscrits dont plusieurs probablement ne donnent pas leurs cours».

37 Décanat, Registre des séances de la Faculté de médecine, nº 1, p. 244, le 29 avril 1885. 
cliniques (voir note 20). C'est déjà une limitation importante de l'idée libérale du départ, motivée sans doute par le danger de voir des institutions privées menacer les institutions d'enseignement et de soins publics. Pourtant, la volonté des professeurs de mieux contrôler l'enseignement théorique est toujours mise en échec. A côté des plaintes régulières, de la volonté réitérée de réviser la loi qui régit les privat-docents et de quelques rares exclusions du corps des privat-docents pour des raisons administratives, deux affaires un peu plus conséquentes permettent de mieux cerner les enjeux autour du statut de privat-docent.

\section{a) L'affaire Geib, publicité déloyale}

La première affaire importante concerne le Dr Geib, docteur en médecine de l'Université de Munich, qui obtient dès l'ouverture de la Faculté, l'autorisation de donner un cours de privat-docent. Quoique le cours du Dr Geib figure au programme, il n'y a pas de trace d'inscriptions prises en son nom. C'est probablement ce défaut d'inscriptions - suggérant l'absence de tout enseignement - qui incite le DIP à demander l'avis de la Faculté de médecine, en juin 1880, sur un cours que ce médecin désire donner le semestre suivant ${ }^{38}$. La Faculté répond que

«(...) considérant que le titre du Cours qu'il désire faire n'a rien de scientifique et ne vise que la réclame, comme prouvent surabondamment ses annonces sur la quatrième page des journaux et ne voulant pas se prêter à des manœuvres de cette nature, [elle] s'oppose à lui donner l'autorisation qu'il demande ${ }^{39}$.»

Une lecture attentive des journaux contemporains n'a fourni qu'une annonce, d'apparence anodine (fig. 1). Il est vrai que le Dr Geib a inséré son annonce à plusieurs reprises entre le 23 janvier et le 5 février $1880^{40}$. Pourtant, son encart ne signale pas son affiliation avec l'Universitée ${ }^{41}$. Les reproches de la Faculté se fondent, à première vue, sur le rapport entre le sujet du cours projeté (Nouveau procédé pour le traitement de la syphilis) et la spécialité revendiquée dans l'annonce. Les procès-verbaux des organes universitaires ne nous ont rien appris de plus, par contre, l'affaire prend un sens différent à la lumière des procès-verbaux de la Société médicale de

38 Ce qui est confirmé par un échange épistolaire entre le Recteur et le DIP. Voir plus haut note 26.

39 Décanat, Registre des séances de la Faculté de médecine, nº 1, p. 134.15 juin 1880.

405 fois dans la Feuille d'Avis, 3 fois dans le Courrier de Genève et 2 fois dans la Tribune de Genève.

41 Une autre publicité, cette fois du Dr Glatz dans le Bottin genevois (1880) semble bien plus abusive. En effet, Glatz se targue du titre de: "professeur libre de balnéothérapie à la Faculté de médecine de Genève.» Une lettre du Recteur au DIP nous apprend que Geib aurait, lui aussi, usurpé le titre de «professeur libre» (AEG, I.P. Q 125-126, 18 octobre 1880). 


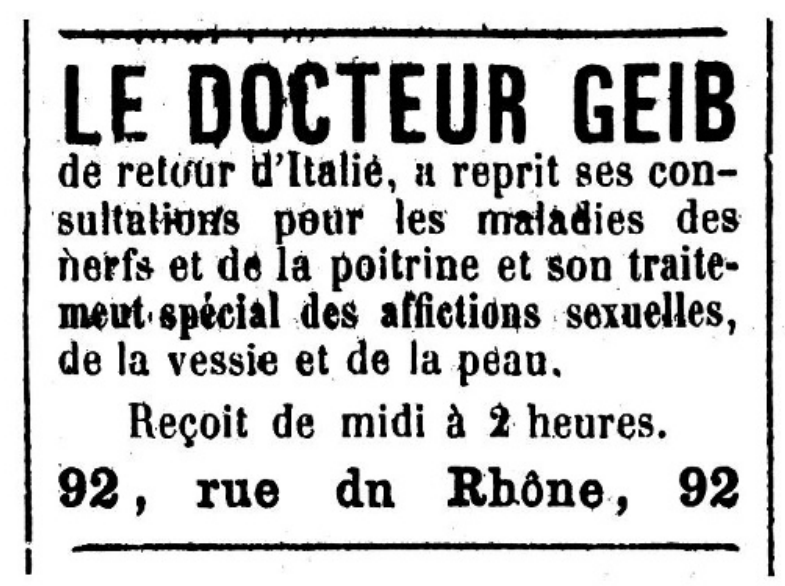

Fig. 1: Courrier de Genève, samedi 31 janvier 1880 .

Genève. Dans la deuxième moitié du XIX ${ }^{\mathrm{e}}$ siècle, cette société se donne le mandat de «surveiller les intérêts du corps médical» corps médical français ${ }^{43}$, la Société médicale combat les «charlatans», en dénonçant, notamment, les réclames dans la presse. Les médecins qui font de la réclame sont ainsi mal vus - ils discréditent le corps médical ${ }^{44}$. Cette campagne aboutit, deux ans avant l'affaire Geib, en 1878, à l'impression d'une brochure par la Société médicale, brochure qui met en garde la population contre les annonces. Le texte, tiré à 5000 exemplaires et distribué gratuitement dans les pharmacies, explique aux patients que «l'expérience nous apprend que, en médecine surtout, toute réclame doit être considérée comme suspecte ${ }^{45}$ ».

L'exclusion de Geib résulte logiquement de cette campagne ${ }^{46}$. La réaction universitaire souligne l'intelligence entre les chefs de file de la Société médicale et les professeurs de la Faculté de médecine. Plusieurs professeurs font partie de cette société et fort de leur prestige, y prennent un rôle prépondérant; en 1880 deux des trois éditeurs de la nouvelle Revue médicale de la Suisse romande sont enseignants à la Faculté. La Société médicale, axée essentiellement sur les échanges scientifiques se voit renforcée par le prestige de compter des universitaires dans son rang; parallèlement, les enseignants

42 Règlement de la Société médicale de Genève, Genève, 1835, art. 2.

43 Cf. LEONARD Jacques, La médecine entre les pouvoirs et les savoirs, Paris, 1981, pp. 170-175.

44 Bulletin de la Société médicale de la Suisse romande, 1871 et 1872. Les deux rapports des présidents pour 1870 et 1871 (respectivement Dr Mercier et Dr Maunoir) mentionnent ce conflit.

45 La Société médicale du canton de Genève et la Société de pharmacie de Genève au Public, Genève, 1878.

46 Une partie de la correspondance avec Geib est conservée; officiellement seule la scientificité de l'enseignement est mise en cause. Seujet, Pièces diverses et correspondance, 1878-1883, lettre du 21 juin 1880. Geib ne figure plus au programme des cours l'année suivante. Cette affaire est à l'origine d'une correspondance entre le Rectorat et le DIP et aura pour effet la mise sur pied du Règlement spécial pour les cours des Privat-docents à l'Université de 1880 (voir p. 80/81). 


\section{$\overline{\text { LeDocteurS.KESER }}$ 438 Privat-Docent à l'Université reçoit de 1 à 3 h., Place da Port, 1. Traitement des états maladifs du sang, des diverses Anémies et des Affections de la peau. Consultations gratuites: \$farché 20, Lundi, Mercredi, Samedi, de 10 à 12 heures.}

Fig. 2: Tribune de Genève, samedi 4 avril 1895.

universitaires y trouvent l'occasion d'asseoir leur primauté sur les médecins établis en ville. On peut ajouter que très peu de temps après cette affaire, le monde médical genevois cède devant la pression de la concurrence et de la publicité. Au cours des années 1890, des membres tout à fait respectables du corps médical genevois placent régulièrement des annonces dans les quotidiens; certains se contentent d'indiquer l'horaire de leur cabinet, d'autres étalent leurs spécialités ${ }^{47}$. Ainsi, déjà en 1895 , le Docteur Keser peut insérer une réclame dans plusieurs journaux genevois qui met en évidence son statut de privat-docent, sans susciter la moindre remarque ${ }^{48}$ (fig. 2).

\section{b) Privat-docent et politique}

La seconde affaire concerne le Dr Alcide Jentzer, connu aujourd'hui surtout pour son rôle dans la construction de la Maternité. Jentzer fut d'abord assistant de Vaucher (obstétrique), puis, dès l'année universitaire 1878-1879, il annonça un cours de privat-docent en gynécologie. Il bénéficia de ce statut pendant plusieurs années. En été 1882, le Bureau de l'Université est averti du fait qu'il ne donne pas son enseignement «régulièrement» et décide de lui en faire la remarque. L'année suivante, le prof. Vaucher écrit une véritable lettre de dénonciation au Recteur:

«(...) M. Jentzer a annoncé ce semestre d'hiver des répétitoires, il a occupé des heures sur le tableau des cours qui auraient peut-être pu être utilisés par d'autres et au $1^{\text {er }}$ janvier il avait terminé, il me semble que tous ceux qui enseignent à l'Université sont soumis aux mêmes obligations, je ne suppose pas qu'il y ait deux poids et deux mesures. Veuillez M. le Recteur faire faire une enquête sur les faits que je vous signale et prendre note de ma réclamation» ${ }^{49}$.

47 Dans la Tribune de Genève de 1895, il y a tous les jours 2 à 3 annonces de ce type. Dans le Courrier et le Genevois, il y en a régulièrement, mais jamais autant que dans la Tribune. Le nombre d'annonces dans la presse ne fera que croître au cours de années suivantes. En 1906, dans la Tribune, il y a quotidiennement entre 8 et 10 encarts publicitaires de médecins; les autres quotidiens connaissent aussi un accroissements, quoique moindre, de petites annonces médicales.

48 Par exemple, Journal de Genève, le 8 janvier 1895.

49 Seujet, Pièces diverses et correspondance, 1883-1888, lettre datée du 13 mars 1883 . Les relations entre Jentzer et Vaucher n'étaient pas très bonnes, comme en témoigne une lettre du 
Ce n'est donc pas à proprement parler par hasard que le 2 juin de la même année (1883), le prof. Julliard (prof. de clinique chirurgicale et Recteur) pose à la Faculté la question de savoir s'il est opportun de donner l'autorisation à Jentzer de "continuer ses Cours à la Faculté de Médecine en qualité de privatdocent». Dans la discussion qui suit, Julliard fait part de ses doutes concernant le grade de Docteur de Jentzer, grade

«dont il n'a jamais cessé de se prévaloir auprès des Recteurs de l’Université, pour obtenir selon la loi, l'autorisation de faire des Cours libres à la Faculté de médecine; il a demandé à M. Jentzer de produire son diplôme de Docteur en médecine (...) la Faculté par un vote régulier déclare à l'unanimité, que les pièces présentées par M. Jentzer ${ }^{50}$, sont absolument incomplètes et ne lui confèrent en aucune façon le grade de Docteur en médecine ${ }^{51}$.»

La Faculté n'arrive pas à se déterminer sur la voie à suivre; à savoir s'il faut insister pour qu'il cesse ses cours ou non. Jentzer est député radical au Grand Conseil (depuis 1880), membre de la Commission administrative de l'Hôpital cantonal (depuis 1882) et compte de nombreux appuis politiques. A noter que ses compétences ne sont jamais remises en question ${ }^{52}$. C'est le $\mathrm{Bu}-$ reau, à qui l'affaire est transmise, qui tranche:

«Le Bureau, à l'unanimité, charge le Recteur d'écrire à M. Jentzer que, dans ces circonstances, l'Université de Genève ne peut pas l'autoriser elle-même à donner un cours de privat-docent. Le Recteur renverra M. Jentzer à les pourvoir devant le Département, pour être autorisé par lui à donner un cours de privat-docent ${ }^{53}$.»

Jentzer suit ce conseil et obtient sans peine l'autorisation nécessaire, le Bureau se contentant de préciser, qu'il «est entendu que sur ce programme universitaire le titre de Docteur n'accompagne pas le nom de M. Jentzer ${ }^{54}$ ». C'est - à ma connaissance - le seul des enseignants en médecine à figurer ainsi sans le titre de docteur; c'est sans doute aussi le seul qui ait reçu l'autorisation de donner un cours à la Faculté de médecine en passant par la voie

Département de Justice et Police au DIP qui fait cas du retard dans les examens des élèves de la maternité, retard imputé au «refus par le professeur Vaucher de siéger avec M. le Docteur Jentzer, pour faire passer les examens de la Maternité» (AEG, fonds DIP, Q 129-130, lettre du 31 octobre 1882).

50 Les documents discutés ne sont pas énumérés explicitement lors de cette séance. Par contre, une séance du Bureau du Sénat du 13 juin 1883 reprend ce problème: «M. Alcide Jentzer (...) a passé en janvier 1874 devant la Faculté de médecine de Würzbourg un bon examen de docteur, et soutenu des thèses; mais il n'a pas fait la dissertation exigée par les Règlements universitaires, en sorte que l'Université de Würzbourg ne lui a pas donné le diplôme de Docteur, qu'il ne doit recevoir qu'après avoir fait la dite dissertation, quoiqu'il soit déjà Docteur rite promotus.»

51 Décanat, Registre des séances de la Faculté de médecine, n 1, pp. 190-191, 12 juin 1883.

$52 \mathrm{Ibid}$, dans la même séance: «M. le Prof. Reverdin prend la parole pour déclarer que tout en reconnaissant que M. Jentzer ne possède pas le Diplôme de Docteur en Médecine, il serait fâcheux de ne pas lui donner l'autorisation de continuer ses Cours libres (...).»

53 Seujet, Bureau du Sénat, 13 juin 1883.

54 Ibid, le 18 juin. 
réservée à ceux qui n'ont pas de diplôme reconnu. De toute façon, le 8 septembre 1883, Jentzer est nommé chargé de cours par le Conseil d'Etat, sans que la Faculté de médecine soit consultée. Notons que l'arrêté de nomination porte la mention «Monsieur» et non «Docteur» ${ }^{55}$ ! Par la suite, Jentzer parviendra à obtenir officiellement le titre de docteur ${ }^{56}$. La nomination de Jentzer souligne l'impuissance de l'Université face aux autorités politiques et éclaire, du moins en partie, les difficultés qu'ont les professeurs à obtenir le contrôle des nominations ${ }^{57}$. Les registres de la Faculté sont peu explicites sur les tensions que génèrent cette situation; la démission de Jentzer quelques années plus tard est officiellement mise au compte des insuffisances du matériel clinique, mais déjà les auteurs de sa nécrologie attribuent cette démission à des tensions politiques ${ }^{58}$. Les cas «Geib» et «Jentzer» cachent une réalité plus anodine, un nombre toujours plus important d'enseignants qui donnent ou ne donnent pas leur cours, sans qu'il y ait de contrôle. La Faculté de médecine prendra progressivement une position plus dynamique face au problème et c'est elle qui aura une influence déterminante sur l'évolution du statut.

\section{La Faculté de médecine et l'évolution du statut de privat-docent (1885-1916)}

\section{a) Les premiers pas}

Depuis l'ouverture de la Faculté, les professeurs de médecine guettent une occasion pour réviser le statut de privat-docent ${ }^{59}$. En 1885, les délibérations autour de la révision de la loi sur l'Instruction publique de 1872 vont leur apparaître comme une opportunité à exploiter. Ironiquement, c'est le député

55 Arrêté du Conseil d'Etat du 8 septembre 1883.

56 Le 16 novembre 1883, le Bureau du Sénat est informé que: «M. le Dr Alcide Jenzter a fini par obtenir de l'Université de Würzbourg, la délivrance de son diplôme, en sorte qu'il est aujourd'hui Docteur, non pas seulement rite promutu, mais vraiment in optima forma.»

57 Par exemple, quelques brochures publiées à l'occasion de la révision de la loi sur l'Instruction Publique (1885-1886):THURY M., Quelques défauts de l'Université de Genève, Mémoire sur plusieurs réformes qu'il serait urgent d'introduire dans le professorat, Genève (Georg), 1885, pp. 5-8; FOL, H., Genève et son Université, Genève, 1886, pp. 13-19; VOGT C., Quelques mots sur la question universitaire, Genève, 1886, p. 57. La lutte de pouvoir entre le DIP et l'Université est bien connue, voir l'historiographie de l'Université.

58 Genevois, le 28 janvier 1907 (auteur anonyme) et Tribune de Genève, le 3 décembre 1946 (signé P. B.).

59 Ce désir de contrôle n'est pas réservé à la Faculté de médecine, mais il est moins persistant dans les autres facultés. 
Jentzer (membre d'une commission parlementaire) qui sollicite l'avis de la Faculté en février 1885, sur le statut des privat-docents. La Faculté décide de réclamer qu'on exige plus de garanties scientifiques de ceux qui désirent devenir privat-docent - résolution qui est répétée à plusieurs reprises ${ }^{60}$. Cette demande ne sera entendue qu'à moitié par les parlementaires qui voient d'un mauvais œil les tentatives de contrôle des professeurs. En effet, dans l'article 137 de la loi de 1886 (5 juin), s'il est vrai que les candidats ayant fait leurs études ailleurs qu'à Genève doivent obtenir l'accord de la Faculté en question (pour la Faculté de médecine, une clause stipule qu'une dissertation spéciale est nécessaire), la possibilité est toujours donnée aux «anciens professeurs, docteurs et licenciés de l'Université de Genève» d'annoncer des cours de privat-docents sans épreuve scientifique supplémentaire ${ }^{61}$. La Faculté a maintenant son mot à dire dans les autorisations données aux candidats ayant fait leurs études ailleurs ${ }^{62}$. Le premier décembre 1886, le Dr Wyss (docteur de Berne) se voit exiger une thèse d'habilitation, c'est le premier ${ }^{63}$. A partir de cette date, tous les diplômés (ou presque ${ }^{64}$ ) d'autres universités devront se plier à cette condition. Pour la petite histoire, notons qu'à partir de cette nouvelle loi sur l'Instruction publique (1886, art. 155), l'Etat encaisse la moitié du casuel des professeurs (nommés après 1886) et des privat-docents. Ces derniers assurent donc un enseignement gratuit et pourvoient aux caisses de l'Etat! C'est sans surprise qu'on constate la réticence du DIP à octroyer l'autorisation d'enseigner gratuitement - l'Etat perd Frs 2.50 par étudiant et par cours ${ }^{65}$ !

La loi de 1886 ne satisfait pas les professeurs de médecine.A partir de 1892, on trouve régulièrement dans les organes universitaires des propositions visant à exiger une dissertation spéciale de tous les candidats au titre de

60 Décanat, Registre des séances de la Faculté de Médecine, n 1, pp. 236-237, le 23 février 1885 (aussi le 3 avril et le 28 mai de la même année).

61 Par ailleurs, l'art. 137 est augmenté comme suit: «Le département, sur préavis du Bureau du Sénat, peut supprimer un cours de privat-docent pour des motifs graves.»

62 Seujet, Bureau du Sénat, 11 novembre 1887:le Bureau approuve le plan proposé par la Faculté de médecine au sujet de l'habilitation de privat-docents. Ce plan veut qu'une fois approuvées, ces thèses soient imprimées à 150 exemplaires et défendus publiquement.

63 Décanat, Registre des séances de la Faculté de médecine, n 1, pp. 295-296, 18 novembre 1886.

64 Des dérogations sont données, par exemple pour le Dr Lardy, qui l'obtient en raison de sa fonction de président de la commission des examens fédéraux (AEG, fonds DIP, 1985 va 5.3.23, Rapport du 5 juin 1908).

65 Seujet, Pièces diverses et correspondance, 1883-1888. En effet, une lettre du 24 oct. 1888 du conseiller d'Etat chargé du DIP informe le Recteur: «J'ai communiqué au Conseil d'Etat la question soulevée par le Bureau du Sénat à propos du droit que lui conférait l'ancien Règlement de l'Université d'autoriser les privat-docents à donner gratuitement certains cours. Après examen du sujet, le Conseil d'Etat a estimé, qu'à l'avenir, MM. les privat-docents qui désireraient donner gratuitement leurs cours devront en demander directement l'autorisation au Département qui statuera sur chaque cas, sur le préavis du Bureau du Sénat.» 
privat-docent, propositions auxquelles il ne sera pas donné de suite ${ }^{66}$. En 1896 au Bureau du Sénat, le professeur Julliard «attire l'attention du Bureau sur le grand nombre de Privat-docents inscrits dont plusieurs probablement ne donnent pas leurs cours». Il propose l'envoi d'une circulaire à tous les privatdocents pour leur rappeler le règlement ${ }^{67}$. Cette démarche correspond bien à une tentative de reprise en main de la situation et nous confirme dans l'hypothèse que le règlement n'est pas appliqué; le Bureau ne sait pas ce qui se passe dans les amphithéâtres et laboratoires! Cette motion vient interrompre le train-train et suggère une application plus stricte des moyens de contrôle à la disposition de l'Université (voir plus haut p. 80/81). L'augmentation régulière du nombre de privat-docents dans la Faculté de médecine depuis 1890 (fig. 3) pourrait être à l'origine de son initiative. L'habitude d'envoyer cette circulaire accompagnée d'un questionnaire se maintient plusieurs années. En 1896, sur 38 réponses, 14 privat-docents avouent ne pas donner leur cours; la situation la plus catastrophique s'observe en médecine où sur 15 enseignants qui répondent à la circulaire, seuls cinq affirment enseigner ${ }^{68}$ ! Vérification faite, il y a 20 cours annoncés dans le programme, alors que les inscriptions prises par le bedeau suggèrent 7 cours donnés, tous avec plus de 5 inscrits, sauf le cours du Dr Binet qui n'en compte que 3: il en reste donc 13 dont l'existence est improbable ${ }^{69}$. Pour le semestre suivant, les proportions sont sensiblement les mêmes ${ }^{70}$. Malgré l'envoi de ces circulaires, l'administration du Bureau est toujours aussi inefficace dans le contexte de la gestion des privat-docents ${ }^{71}$. Devant le manque d'efficacité des démarches effectuées, des initiatives plus discrètes sont avancées, comme celle du Prof. Eternod qui suggère tout simplement, en février 1902, de faire disparaître les cours des privat-docents du programme des cours ${ }^{72} \ldots$

$\mathrm{Au}$ sein du corps des enseignants libres, il y a maintenant un nombre de plus en plus important d'anciens étudiants et d'anciens assistants de la Faculté elle-même - ce qui rend la disposition de la loi de 1886 encore plus futile. Il

66 Par exemple Décanat, Registre des séances de la Faculté de médecine, ${ }^{\circ}{ }^{2}$, le 19 décembre 1892.

67 Ibid, 16 mai 1896.

$68 \mathrm{Ibid}, 6$ juin 1896.

69 Seujet, Inscriptions aux cours en médecine. Le plus populaire compte 20 étudiants et est donné par le Dr Martin sur les maladies de l'enfant (en concurrence avec le cours du prof. d'Espine). Les autres comptent entre 6 et 16 inscrits.

70 Seujet, Bureau du Sénat, 12 décembre 1896.

71 Par exemple, Bureau du Sénat, 19 mai 1899: un nouveau circulaire est envoyé en 1899, «pour leur demander de s'inscrire au programme du semestre prochain. Ceux qui ne répondront pas seront biffés». Les réponses sont communiquées le 11 janvier 1901, mais le détail ne figure pas au procès-verbal et il n'y a pas de trace de la sanction prévue.

72 Ibid, 28 février 1902. 


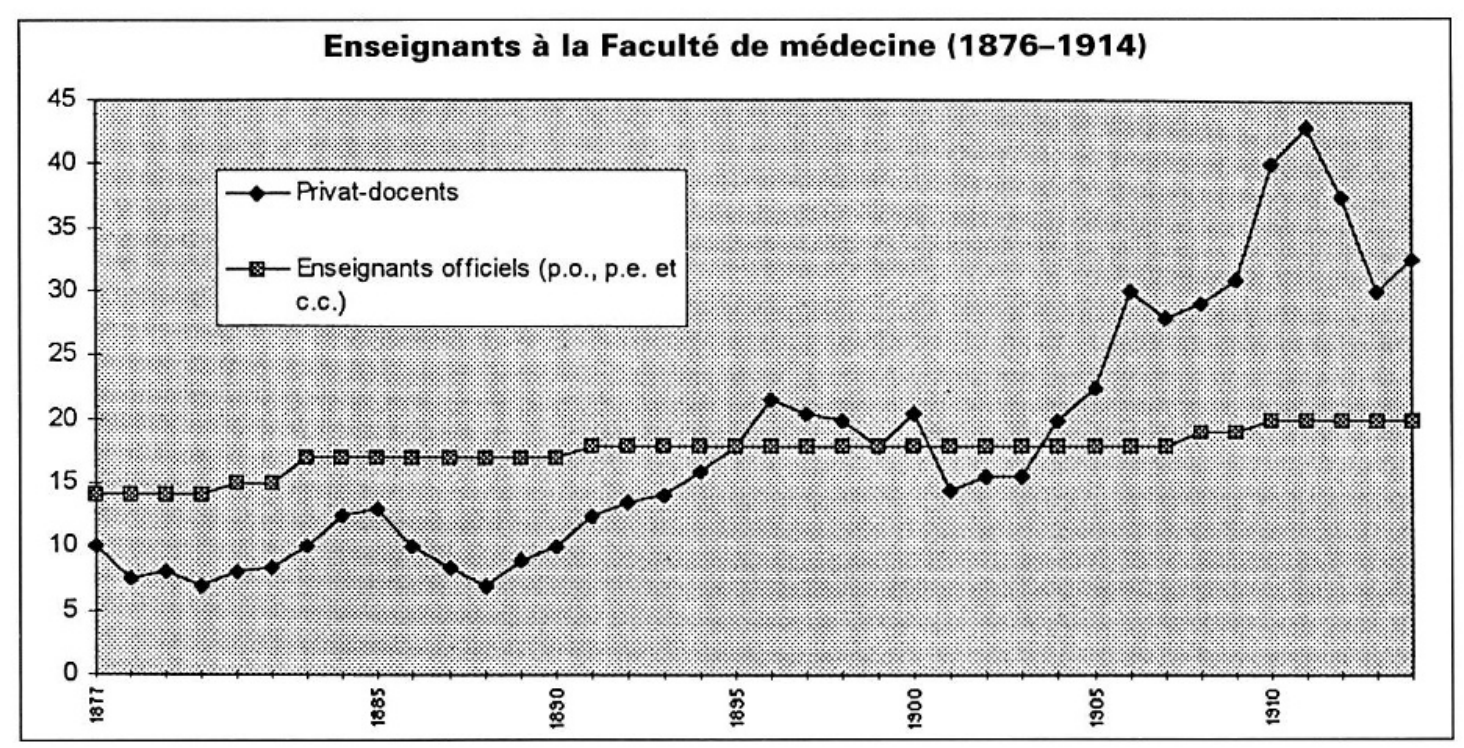

Fig. 3

n'y a pas de règle apparente pour le succès ou l'insuccès d'un cours. Toujours d'après les registres d'inscriptions, signalons que la popularité touche aussi bien de cours théoriques (en 1896, celui du Dr Kummer [chirurgie] et celui donné par le Dr Thomas [diagnostic médical]), que des cours pratiques (toujours en 1896, la clinique infantile du Dr Martin connaît un grand succès). Tout laisse entendre que les ingrédients essentiels pour la réussite d'un enseignement sont la pertinence du sujet par rapport aux examens fédéraux et la collaboration du privat-docent avec le professeur titulaire ${ }^{73}$. Certaines collaborations vont très loin, comme celle du Professeur Weber (psychiatrie), qui demande à se décharger d'une partie de son enseignement théorique en faveur de son assistant, privat-docent ${ }^{74}$.

Dans le contexte du début du siècle et en dépit des abus, l'encadrement très souple d'enseignants et cliniciens «libres» fut un soutien indispensable à la bonne marche de la Faculté. Alors que le nombre d'étudiants augmentait de façon presque dramatique, que celui des titulaires stagnait et que les locaux devenaient insuffisants, les privat-docents ont assuré une charge d'enseignement toujours croissante, allant parfois jusqu'à fournir le matériel clinique indispensable ${ }^{75}$. Il est donc indéniable que les prestations des privat-docents ont permis des économies à l'Etat et assuré la croissance des effectifs dans un contexte économique et budgétaire plutôt sombre.

73 Ce qui est confirmé par les travaux de Marcacci, MARCACCI Marco, op. cit, 1987, p. 49.

74 Seujet, Bureau du Sénat, 26 octobre 1905. La crainte du Bureau est de voir ce cas se généraliser ...

75 Tout comme les privat-docents allemands: BUSCH Alexander, op. cit., pp. 319-341. 


\section{b) La Faculté prend l'affaire en main}

On peut attribuer une nouvelle initiative de la Faculté de médecine à la croissance linéaire du nombre de privat-docents (voir graphique, fig. 3) au début du siècle. C'est le 7 mai 1904, que le prof. Bard (clinique médicale) réitère une nouvelle fois la proposition d'instaurer une véritable habilitation au cours d'une séance de la Faculté de médecine ${ }^{76}$. Après discussion, une commission est nommée. Cette commission rapporte le 25 juin 1904; l'accueil est positif, et cette initiative de la Faculté de médecine est à l'origine de la création d'une commission universitaire représentant toutes les Facultés, commission dont le rapport fut publié ${ }^{77}$. La majorité de la commission (comprenant les professeurs de médecine, D'Espine et Mayor) propose l'obligation de passer une épreuve devant la Faculté concernée avant d'obtenir le statut de privat-docent (elle prévoit la conservation, indépendante, de cours libres), cette réorganisation est retenue par le Sénat qui décide la modification en fonction du règlement interne de l'Université ${ }^{78}$. Pourtant, à la fin de l'année 1906, l'Université attend toujours une confirmation du DIP ${ }^{79}$. Le retard est dû à la Commission scolaire, laquelle finit par empêcher l'aboutissement de la réforme proposée. Outre un conflit interne d'ordre idéologique (certains désirent maintenir le caractère «libéral» de la loi), les membres de cette commission sont conscients de l'inconvénient du système en vigueur. Devant les propositions d'effacer l'ambiguité soit en supprimant la désignation de «privat-docent», soit en renforçant le privat-docent par une habilitation et en créant, parallèlement, un statut de professeur libre, les avis sont partagés. Finalement, dans sa séance du 19 mai 1907, la commission adopte la résolution suivante:

«reconnaissant les difficultés de principe que susciterait le nouvel article 138, et estimant que les questions soulevées par les propositions du Sénat seraient certainement reprises lors de la discussion d'ensemble sur la loi sur l'Instruction publique, propose à la commission scolaire l'ajournement à terme des propositions du Sénat ${ }^{80} . »$

76 Décanat, Registres de la Faculté de médecine, t. 4,7 mai 1904.

77 Rapports de la Majorité et de la Minorité de la Commission dite des «Privat-Docents», présentés au Sénat dans sa séance du 30 novembre 1905, Genève, 1906.

78 En avril 1906, Alfred Martin (recteur) écrit une lettre au DIP pour faire part de la décision qui venait d'être prise au Sénat (AEG, fonds DIP, 1985 va 5.3.20, [Université/Organisation générale]). Les propositions de modifications sont essentiellement motivées par l'argumentation établie par la Faculté de médecine: «La Faculté de médecine surtout fait observer que, le titre de privat-docent pouvant avoir, aux yeux du public une certaine valeur du point de vue professionnel, des abus graves se sont produits, et risquent de se produire toujours d'avantage.»

79 AEG, fonds DIP, 1985 va 5. 3. 20, (Université/Organisation générale): lettre du Recteur au DIP.

80 AEG, Procès-verbaux de la Commission scolaire, 8 mai 1903-3 juin 1918. Les membres de la sous-commission sont: Bouvier (recteur), Jung (professeur), Duparc, Gautier, Seitz, Guye, Sigg, Bérard. 
Rien ne change. Au semestre d'hiver 1907-1908, plus de la moitié des cours de privat-docents au programme n'ont pas un seul étudiant inscrit. Au semestre d'été 1914, à la veille de la nouvelle loi sur l'Instruction publique, il y a en tout 39 cours de privat-docents annoncés en médecine. Un dépouillement des registres d'inscriptions révèle que seuls 17 comptent plus de cinq étudiants inscrits, 12 ne comptent que quatre étudiants (2) ou moins (10) et sont donc relativement précaires ${ }^{81}$. Il reste 10 cours qui, s'ils ne sont pas suivis par des hordes d'étudiants d'autres facultés, n'ont certainement pas lieu ${ }^{82}$. Il faut attendre la loi sur l'Instruction publique de 1914 pour une première modification; la situation de la Faculté de médecine y est particulièrement intéressante, l'article 263 stipule qu'une dissertation spéciale est exigée des candidats privat-docents dans la Faculté de médecine et que «des conditions spéciales sont fixées par le Règlement de l'Université». La Faculté ne s'inquiétera de clarifier ces «conditions spéciales» qu'en 1916. Le rapport de la commission nommée à cet effet est lue, puis adoptée, à la séance de la Faculté de médecine du 25 novembre 1916. Deux arguments clefs sont avancés. Le premier est que le fait d'octroyer ce titre sans épreuves

«a pour résultat $(\ldots)$ de ravaler notre institution d'instruction supérieure au rang de ces Universités qui distribuent généreusement et sans contrôle, les titres et les grades.»

Le second invoque la responsabilité de la Faculté vis-à-vis du public:

«Elle ne peut oublier qu'Ecole scientifique, elle est aussi, et obligatoirement, Ecole professionnelle, et que rien de ce qui a trait à la profession médicale ne peut lui demeurer étranger. Or si, au point de vue universitaire, le titre de privat-docent de Genève n'a pas, dans l'état actuel des choses, d'autre valeur que celle que lui confère l'homme qui le porte, il n'en est pas de même au point de vue professionnel. Pour le public, ce titre peut encore donner l'impression d'une sorte de distinction qui devient une recommandation. Cette distinction, accordée sans discernement, risque de profiter simplement au plus habile, non toujours au plus digne.»

La Commission conclut par le besoin d'introduire une procédure (comprenant l'examen du dossier du candidat) qui doit mener à de véritables épreuves d'habilitation, pour assurer ce que la commission qualifie de «garanties d'ordre moral comme de l'ordre scientifique» ${ }^{83}$.

81 Dont le cours d'Histoire de la médecine du renommé Charles Greene Custom (1 étudiant inscrit).

82 Seujet, Inscriptions au cours en médecine, hiver 1907-1908, et été 1914.

83 Rapport sur les conditions d'admission au titre de Privat-docent, Genève, 1916. 


\section{Un privat-docent presque parfait}

Le règlement proposé est adopté par le Conseil d'Etat le 5 juin $1917^{84}$. Ainsi, plus de 40 ans après la création de la Faculté de médecine, le statut original de privat-docent, conçu pour encourager les «médecins en ville» à venir renforcer le corps professoral, est amendé pour être aligné sur ce qui se pratique dans les autres universités de tradition allemande ${ }^{85}$. Tous les candidats devront présenter une véritable habilitation.

Rétrospectivement, il est tentant de s'interroger sur les motivations qui ont poussé les professeurs de médecine à insister avec une telle persévérance afin d'obtenir une véritable habilitation. Un meilleur contrôle administratif aurait permis d'éviter les abus les plus flagrants, c'est-à-dire les privatdocents qui profitaient du titre sans donner de cours. Pourtant, les registres laissent percevoir à la base une conception différente de l'organisation de l'enseignement. Les professeurs voient dans les privat-docents un apport complémentaire à leur propre enseignement, alors que les parlementaires rêvent d'un système où régnerait la concurrence. Cette dernière idée est à l'origine de plusieurs défauts indéniables du système imaginé en 1876: le jugement des étudiants ne peut être le seul moyen pour trier les bons des mauvais enseignants; laisser libre champ à n'importe qui d'enseigner semble peu judicieux dans le contexte des progrès scientifiques et de la complexification croissante des connaissances médicales au cap du XX $\mathrm{X}^{\mathrm{e}}$ siècle. S'il n'y a pas de contrôle, comment garantir la formation du médecin?

Le combat mené par la Faculté de médecine aura pour effet principal de démonter un des artifices inventés par les partisans du «projet Université» en 1872 dans le but de vendre l'idée d'une université bon marché aux parlementaires. Il s'agit donc essentiellement de l'échec d'une conception libérale de l'enseignement. Le nouveau contrôle qu'exerce la Faculté a aussi pour effet d'affermir l'autorité de la Faculté sur les médecins établis en ville, médecins qui ne collaborent plus de la même manière, ni surtout, avec la même facilité, à l'enseignement. Il serait néanmoins erroné de conclure sur une note uniformément négative. De nombreux cours ont eu lieu, l'encadrement des étudiants, même lorsqu'ils furent très nombreux, a été assuré. On peut même signaler que, en retranchant les 5 étrangers, 15 des 17 professeurs

84 Règlement d'admission des Privat-docents à la Faculté de Médecine, Arrêté du Conseil d'Etat du 5 juin 1917.

85 La Faculté de médecine de Lausanne semble avoir eu des problèmes similaires. Seulement, comme nous l'avons vu plus haut, la loi de 1890 est déjà moins libérale (cf. note 7) et la Faculté de médecine lausannoise obtient de véritables examens d'habilitations pour les privatdocents déjà en 1909: TISSOT Laurent, Politique, société et enseignement supérieur dans le Canton de Vaud (1890-1916), p. 157. 
nommés à la Faculté de médecine pendant cette période avaient été privatdocents $^{86}$. Même dans sa conception genevoise, le privat-docent est la première marche vers la carrière académique. Il est vrai que ces chiffres sont dérisoires face aux 122 privat-docents qui ont œuvré dans la Faculté de médecine genevoise pendant le même laps de temps (seuls 7 d'entr'eux deviendront professeurs à Genève après 1917).

Une voix, et non la moindre, est absente de cette histoire; c'est celle du privat-docent. On retrouve les enseignants libres sollicitant individuellement l'accès au statut, négociant le titre de leur enseignement, leurs absences, etc. Pourtant, en tant que privat-docents, ils ne font pas partie des organes universitaires, ils ne participent pas aux discussions. C'est toujours à titre individuel qu'ils agissent ${ }^{87}$. Plusieurs privat-docents ont des responsabilités politiques, il serait intéressant de chercher plus loin leur influence (s'il y en a une) sur les autorités politiques dans leurs rapports avec la Faculté, surtout après la législation de fin de siècle qui exclut les fonctionnaires (et donc les professeurs), du Grand Conseil. L'histoire ne s'arrête pas en 1917, la nouvelle codification ne parvient pas à limiter définitivement le nombre de privatdocents; on trouve, par exemple, encore 29 privat-docents inscrits au semestre d'été $1929^{88}$. Somme tout, la situation avant et après la réglementation de 1917 est très similaire à celle que connaît l'Allemagne à la même époque (voir note 3): un nombre important de privat-docents gère une part toujours grandissante de l'enseignement, alors que le nombre d'enseignants nommés n'évolue pas en fonction des charges d'enseignement - autre problème que devra résoudre la Faculté.

86 En 1901, lors de la procédure de nomination du professeur d'Hygiène, il est intéressant de noter que le succès des cours de privat-docents est discuté. D'Espine a fait dresser une liste chronologique des inscriptions, il est vrai que malgré le constat que Marignac a plus d'étudiants que Cristiani, c'est ce-dernier qui obtient le poste. AEG (Terrassière) 1985 va 5.3.23 (Médecine 5), Séance de la commission pour la nomination à la chaire d'hygiène (21/1/1901).

87 Signalons cependant une réunion de privat-docents en 1904, auquel le Prof. Chodat était convié; Seujet, Bureau du Sénat, 6 juillet 1904. Bernard Bouvier l'évoque, lorsqu'il défend devant la Commission scolaire, l'idée d'une véritable habilitation pour les privat-docents. AEG, Procès-verbaux de la Commission scolaire 8 mai 1903-3 juin 1918, 31 mai 1907: «Du reste nos privat-docents se sentent-ils menacés par la proposition d'habilitation? Nullement, puisque, il y a trois sans, ils avaient étudié la question et voulaient la demander.»

88 BUJARD Eugène, «La Faculté de médecine de 1914 à 1956» in Historique de Facultés et instituts de 1914 à 1956, Genève, 1959, p. 230. 